Supporting Information

\title{
Determination of Arsenic Content in Water Using a Silver Coordination Polymer
}

\author{
Natasha D. Reich, ${ }^{\dagger}$ Athena A. Nghiem, ${ }^{\ddagger}, \#$ Sarah Nicholas,,$\S$ \\ Benjamin C. Bostick, ${ }^{\ddagger}$ and Michael G. Campbell ${ }^{*, \dagger}$ \\ †Dept. of Chemistry, Barnard College, 3009 Broadway, New York, NY 10027, USA \\ ‡Lamont-Doherty Earth Observatory, Columbia University, Palisades, NY 10964, USA \\ ${ }^{\#}$ Dept. of Earth and Environmental Sciences, Columbia University, New York, NY 10027, USA \\ §Brookhaven National Laboratory, NSLS-II, Upton, NY 11973, USA
}

*E-mail: mcampbel@barnard.edu 


\section{Table of Contents}

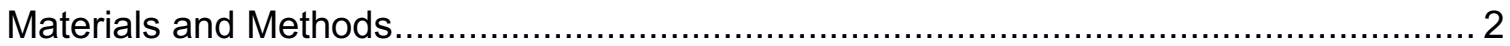

Synthesis of Ag-BTC and Fabrication of Test Strips............................................... 2

Arsenic Testing Procedure for Water Samples ........................................................... 3

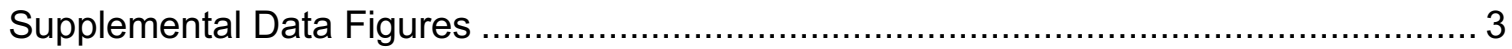

Figure S1. Light Sensitivity of Ag-BTC as compared to $\mathrm{AgNO}_{3} \ldots \ldots \ldots \ldots \ldots \ldots \ldots \ldots \ldots$

Figure S2. Representative images of Ag-BTC strips ........................................... 4

Figure S3. Digital quantification of color change response. ................................ 5

Figure S4. Field testing of a groundwater sample in Cambodia (500 ppb) .............. 5

Figure S5. Field testing of a groundwater sample in Cambodia (40 ppb) ................. 6

Figure S6. PXRD for an Ag-BTC strip before and after testing ............................. 7

Figure S7. XPS spectra for an Ag-BTC strip before and after testing ..................... 8

Figure S8. XFM microprobe map of an Ag-BTC strip after testing .......................... 9

Figure S9. As K-edge XANES for Ag-BTC strips after testing .............................. 9

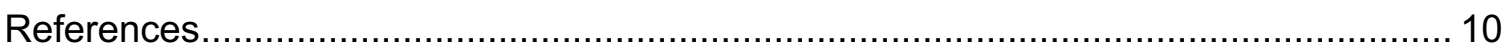




\section{Materials and Methods}

General. All manipulations were carried out under ambient conditions unless otherwise noted. Solvents and reagents were purchased from Fisher and used as received, except for trimesic acid $\left(\mathrm{H}_{3} \mathrm{btc}\right)$, which was purchased from Sigma-Aldrich.

Powder X-ray Diffraction. PXRD data were recorded on a PANalytical XPert3 diffractometer with a $\mathrm{Cu} \mathrm{K} \alpha \mathrm{X}$-ray source and PIXcel 1d detector, in a Bragg-Brentano geometry.

Spectroscopy. X-ray photoelectron spectroscopy data were collected on a PHI 5500 XPS system in the Shared Materials Characterization Laboratory at Columbia University. X-ray fluorescence microprobe (XFM) mapping and As K-edge micro-X-ray absorption near edge-structure ( $\mu$ XANES) spectra were acquired at the XFM beamline (4-BM) at the National Synchrotron Light Source II (NSLS-II), using Kirkpatrick-Baez (KB) mirrors to deliver focused X-rays to the sample. A Canberra SXD 7-element SDD with Quantum Detectors Xspress3 electronics was used, with a Si(111) double-crystal monochromator to control the incident energy for spectroscopy and imaging. The detector was positioned $90^{\circ}$ to the incident beam and the sample was mounted at $45^{\circ}$ to both the detector and the incident beam. Test strips were mounted with Kapton tape onto a sample holder. Arsenate in topaz was used for reference and calibration. XANES spectra were processed in ATHENA, ${ }^{1}$ and XFM maps were processed using SMAK. ${ }^{2}$

\section{Synthesis of Ag-BTC and Fabrication of Test Strips}

Synthesis. We have adapted the literature procedure ${ }^{3}$ in order to synthesize Ag-BTC on larger scale as a phase-pure bulk solid. $2.65 \mathrm{~g}$ of trimesic acid was dissolved in $250 \mathrm{~mL}$ of water, with stirring at $80^{\circ} \mathrm{C}$ until fully dissolved; the solution was then cooled to room temperature. In a separate container, $4.15 \mathrm{~g}$ of silver nitrate was stirred in $125 \mathrm{~mL}$ of $\mathrm{MeOH}$ until fully dissolved, with the container wrapped in foil to minimize light exposure. The two solutions were then combined, resulting in the immediate formation of a cloudy suspension. The mixture was stirred, with the container wrapped in foil, for 12 hours. The solid product was then collected by vacuum filtration, washed with copious water followed by methanol to remove any unreacted starting materials, and dried under vacuum to afford $4.34 \mathrm{~g}$ of Ag-BTC. PXRD analysis was used to assess identity, phasepurity, and long-term stability by comparison with the known structure determined by single crystal diffraction (CCDC 166791).

Fabrication of Test Strips. $7 \times 0.5 \mathrm{~cm}$ strips were cut from sheets of heavy-weight plastic transparency films; the strips were washed with water and then dried. To one end of each strip was applied a $0.5 \mathrm{~cm}^{2}$ piece of double-sided tape. Ag-BTC was ground into a fine powder using a mortar and pestle, and the adhesive end of each strip was then pressed into the powder, shaking gently to remove any excess powder, and repeating if needed until the adhesive area was well coated with a fine layer of Ag-BTC. The test strips were then stored under ambient conditions until use; for long-term storage and transportation the strips were stored in an opaque container to minimize light exposure. Photos of the test strips can be seen in Figure S2 below. 


\section{Arsenic Testing Procedure for Water Samples}

There are a number of commercially available field-test kits for total inorganic arsenic content in water samples based on modifications of the Gutzeit test with mercuric bromide test strips. Two of the most commonly used are from $\mathrm{Hach}^{4}$ and Industrial Test Systems. ${ }^{5}$

In our studies, we adapted the "Econo-Quick" arsenic test kit from Industrial Test Systems (Part No. 481298), which uses a combination of zinc powder and tartaric acid to generate arsine from arsenate and arsenite ions in water. For lab tests a series of arsenic standard solutions ranging from $10-500 \mathrm{ppb}$ arsenic were prepared by serial dilution from a 5 ppm stock solution made from sodium arsenate, using Milli- $Q$ water. The included instructions for the test kit were followed $(50 \mathrm{~mL}$ aliquots of water samples, 10 min exposure time to the generated arsine gas), ${ }^{6}$ except that the included mercurybased test strips were replaced with the homemade Ag-BTC test strips. We note that use of the "second reagent" (potassium peroxymonosulfate), intended to oxidize sulfide impurities, was not necessary for testing of the standard solutions.

\section{Supplemental Data Figures}

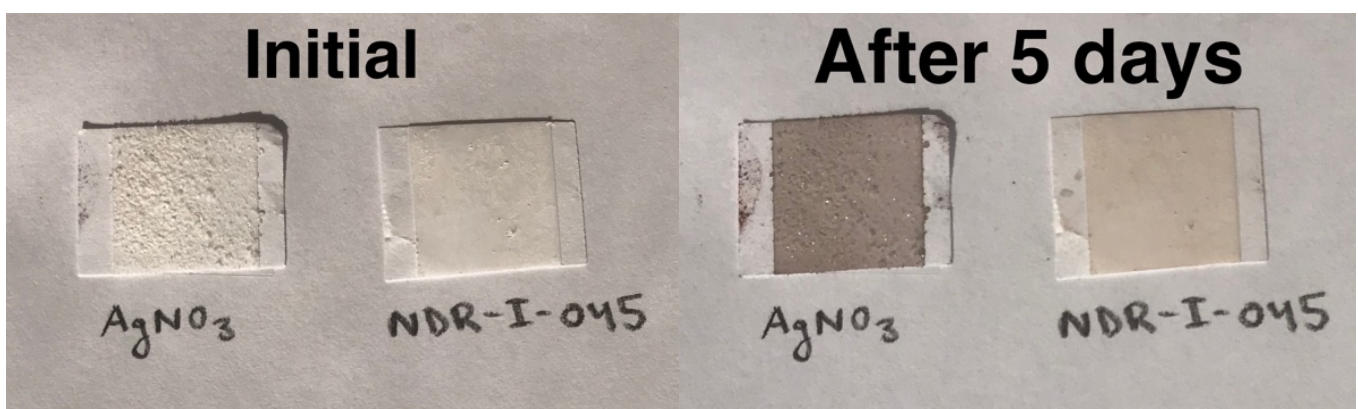

Figure S1. Light Sensitivity of Ag-BTC as compared to $\mathrm{AgNO}_{3}$. Samples of both were finely ground using a mortar and pestle, then applied to a square of double-sided tape on white paper as a backing. The materials were placed on a windowsill that receives direct sunlight and monitored. Discoloration of Ag-BTC was first apparent on day 5, at which point $\mathrm{AgNO}_{3}$ was substantially darkened. 


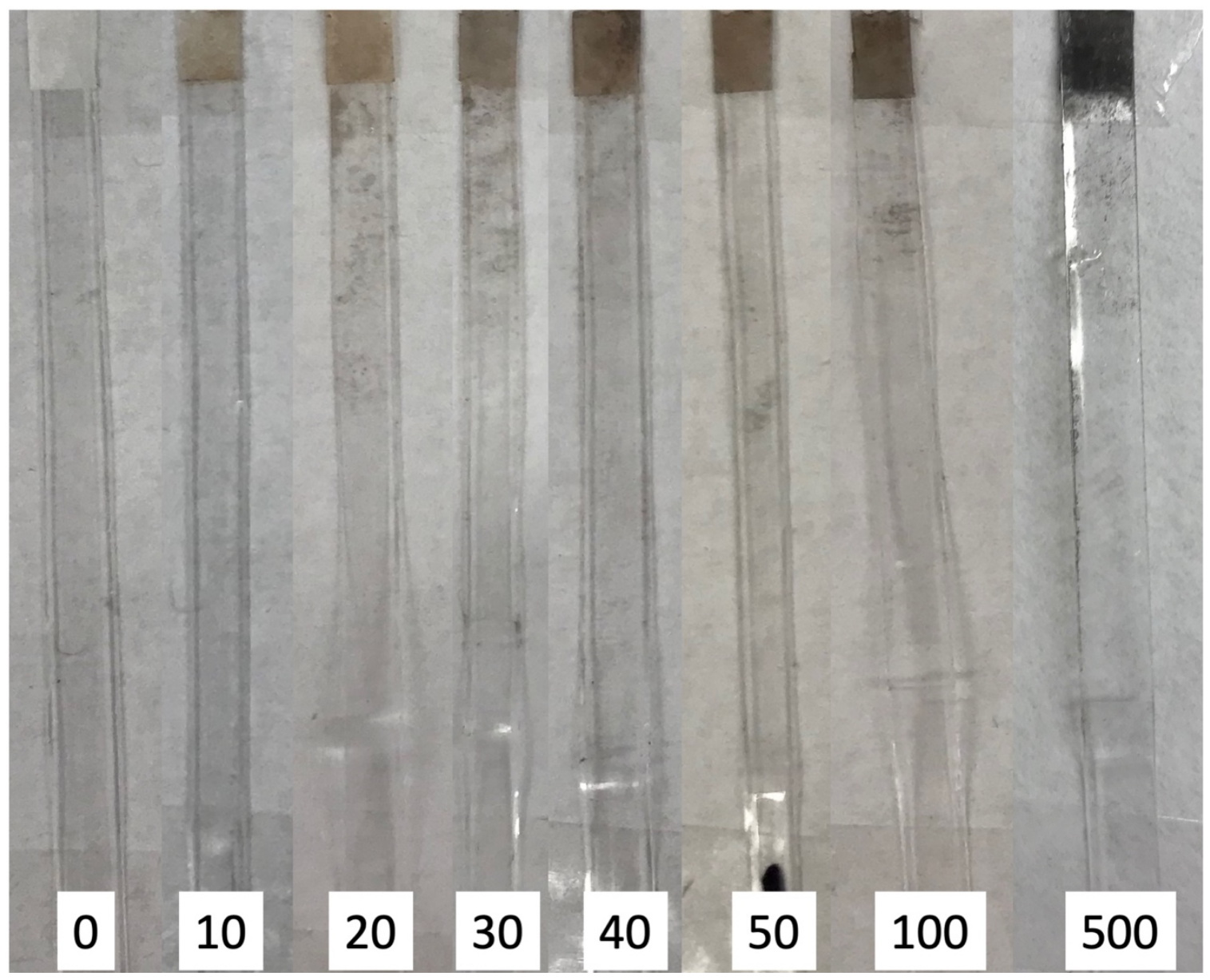

Figure S2. Representative images of Ag-BTC strips after testing water samples with arsenic concentrations ranging from $10-500 \mathrm{ppb}$. The sample with $0 \mathrm{ppb}$ was a control test performed with Milli-Q water. 


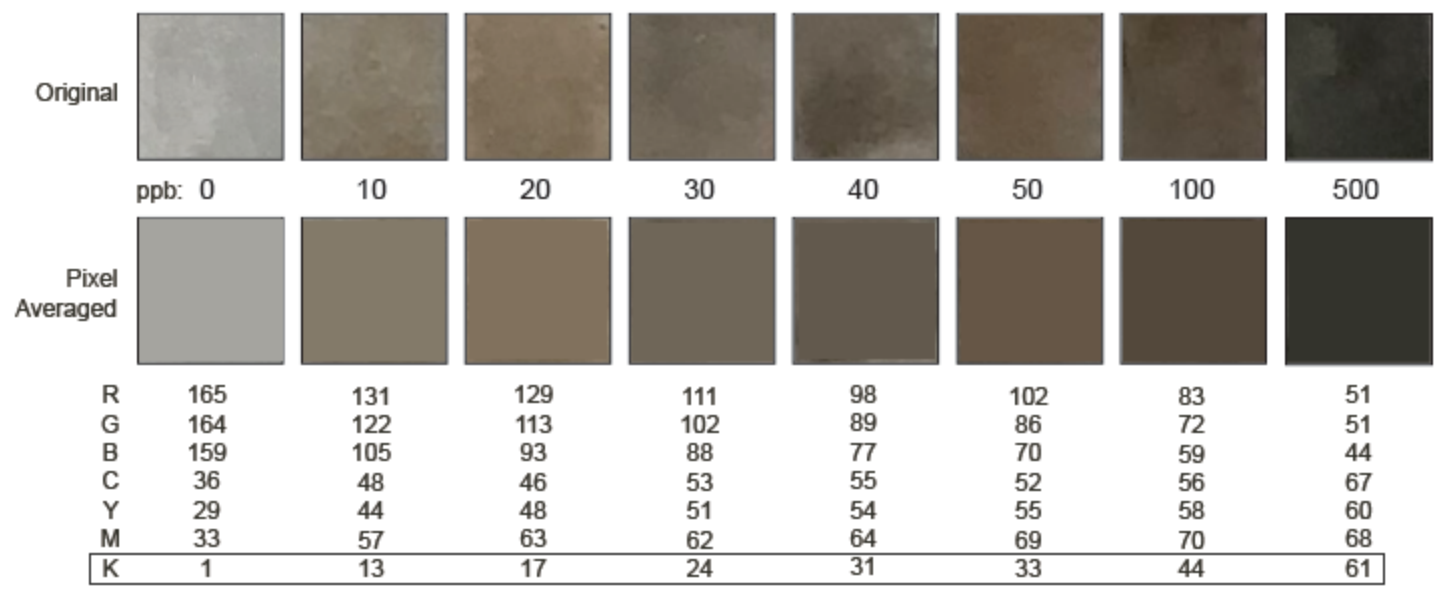

Figure S3. Digital quantification of color change response. The raw images of the test strips were cropped down to the square area containing Ag-BTC (top), pixel averaged (bottom), and then the component RGB and CYMK color values were extracted. The average $\mathrm{K}$ values were then used for the plot of response vs. concentration shown in Figure 3 of the main manuscript.

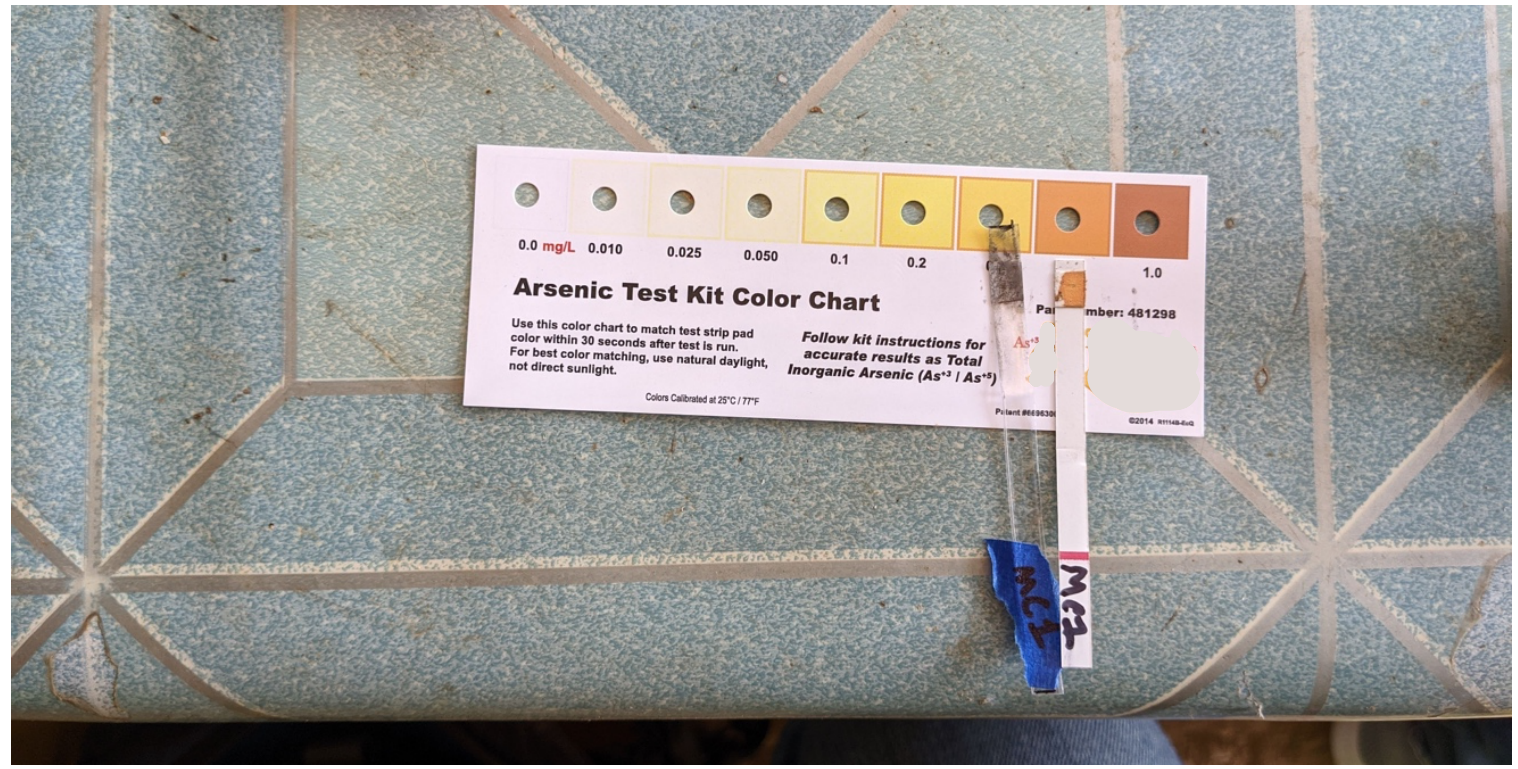

Figure S4. Field testing of a groundwater sample in Cambodia with approximately 500 ppb arsenic concentration, using both an Ag-BTC test strip (clear backing) and a commercial mercury-based test strip (white backing). Identical testing procedures were used for both strips. Collection site location: 11.510233, 105.069738 (degrees northing and easting). Water sample $\mathrm{pH}: 7.24$. Water sample redox status: $+36 \mathrm{mV}$. 


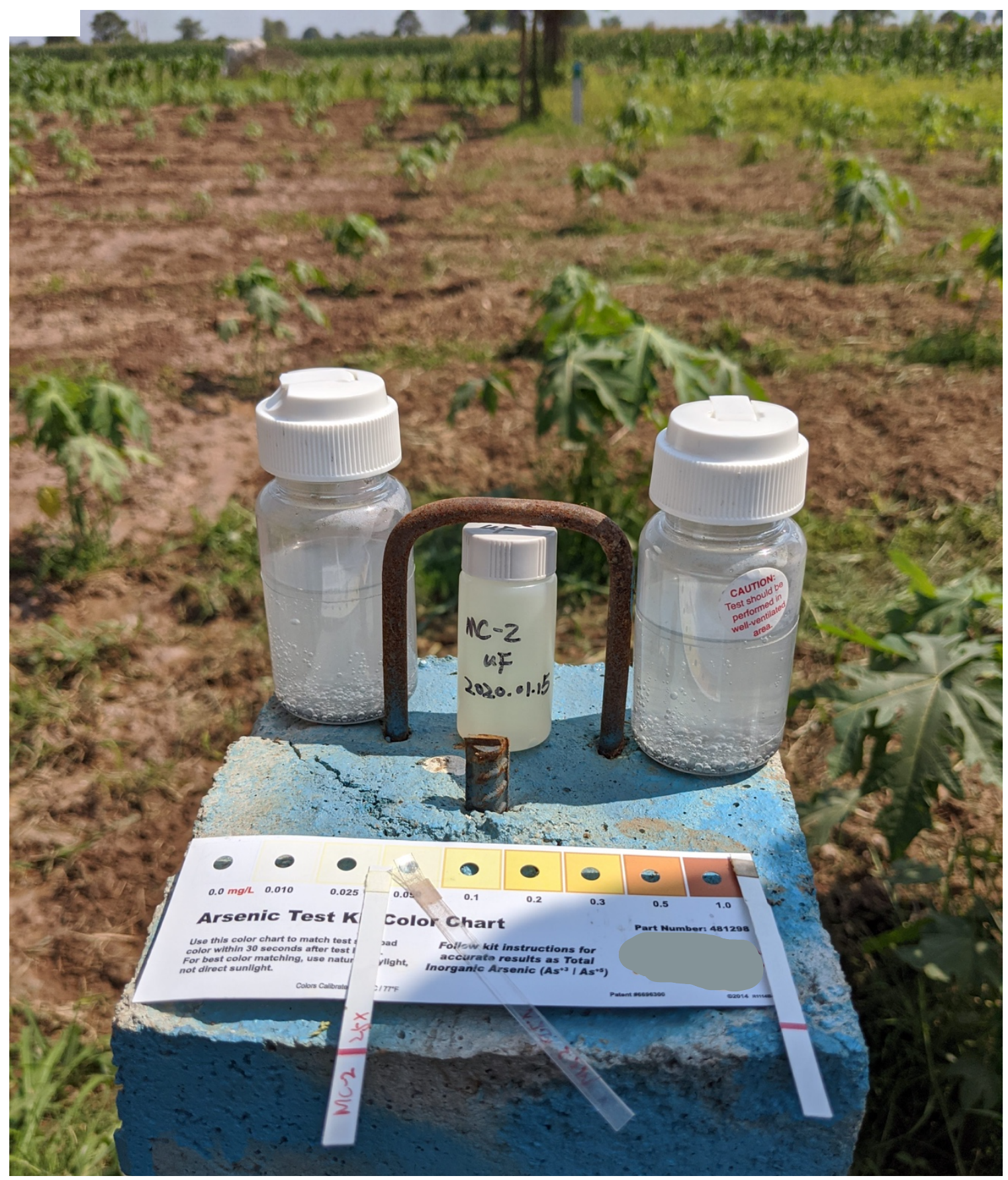

Figure S5. Field testing of a groundwater sample in Cambodia using both an Ag-BTC test strip (clear backing) and a commercial mercury-based test strip (white backing). Identical testing procedures were used for both strips. The water sample initially contained $>1000 \mathrm{ppb}$ arsenic (far right test strip), and was then diluted $25 \mathrm{x}$ prior to measurement with the Ag-BTC strip (left). Collection site location: 11.5205770, 105.0505031 (degrees northing and easting). Water sample pH: 7.25. Water sample redox status: $-70 \mathrm{mV}$. 


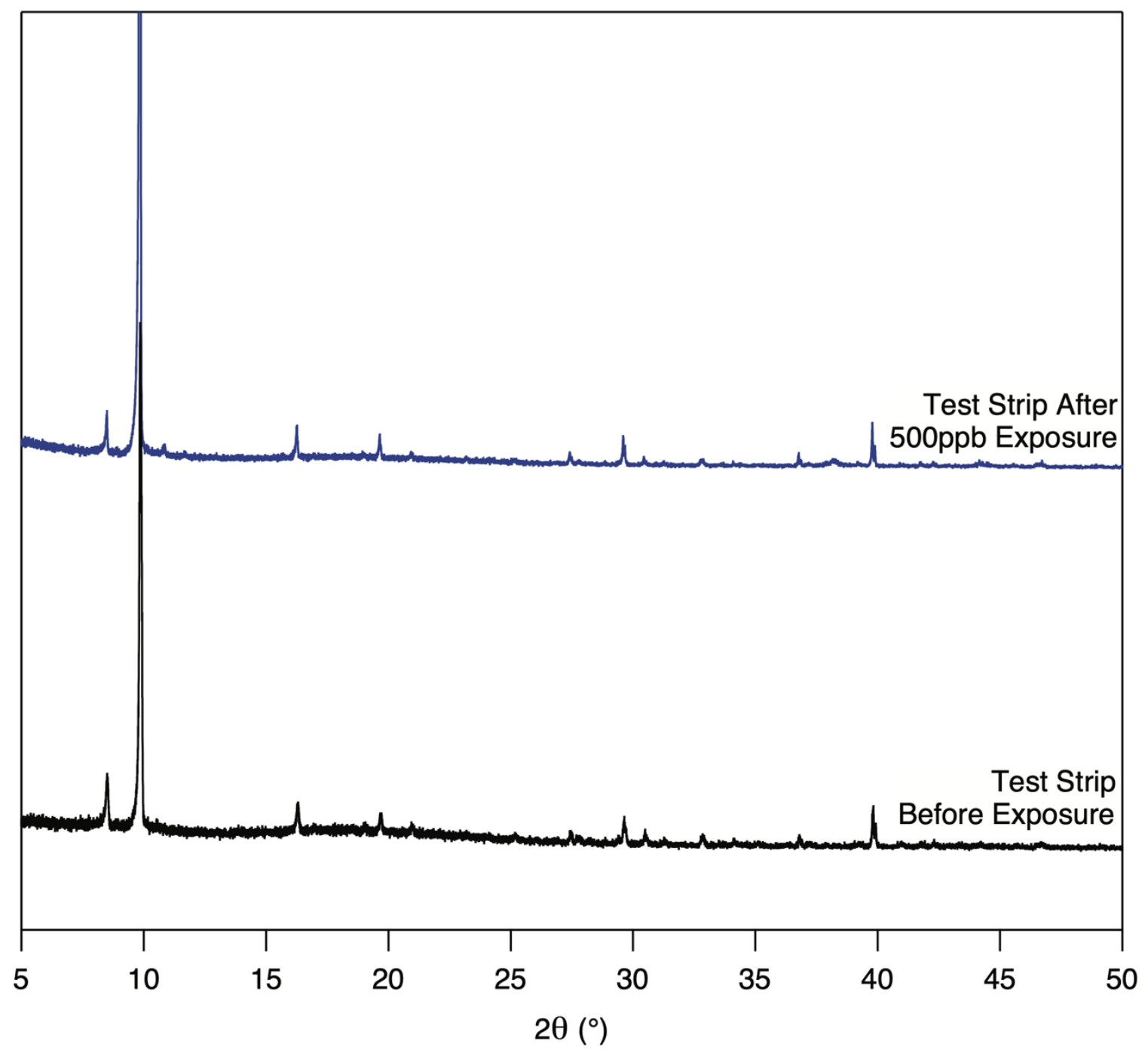

Figure S6. PXRD patterns for an Ag-BTC strip before and after testing a $500 \mathrm{ppb}$ arsenic solution. Despite the small amount of material present, leading to poor signal/noise, the expected peaks for Ag-BTC are clearly visible, and no change is observed after reaction with arsine. PXRD measurements on test strips consistently show a significantly stronger [004] peak as compared to the other reflections, which may be due to preferred orientation effects. 


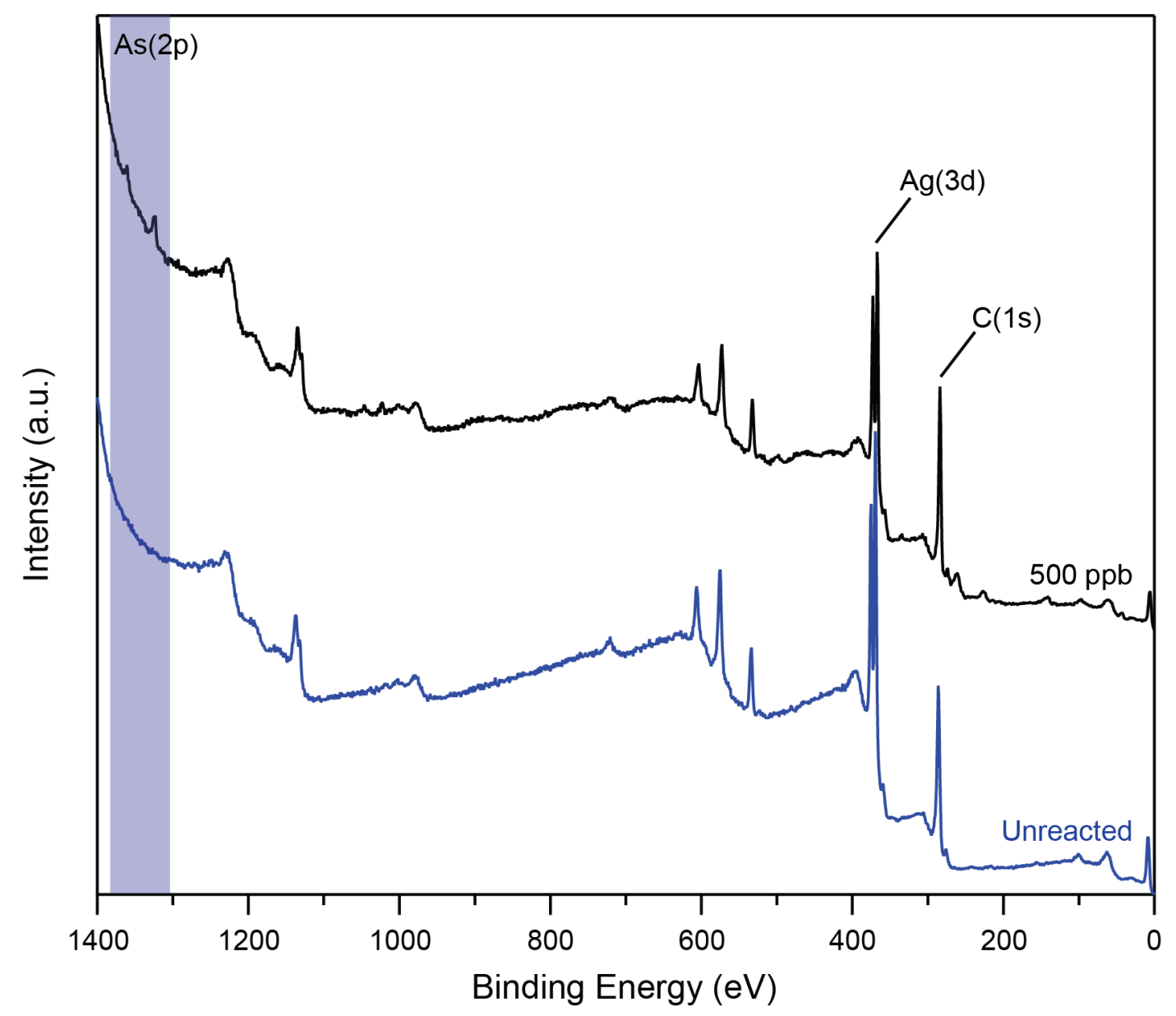

Figure S7. XPS spectra for an Ag-BTC strip before and after testing a 500 ppb arsenic solution. While new peaks appear in the $A s(2 p)$ region after reaction with arsine, no other significant changes were observed. 


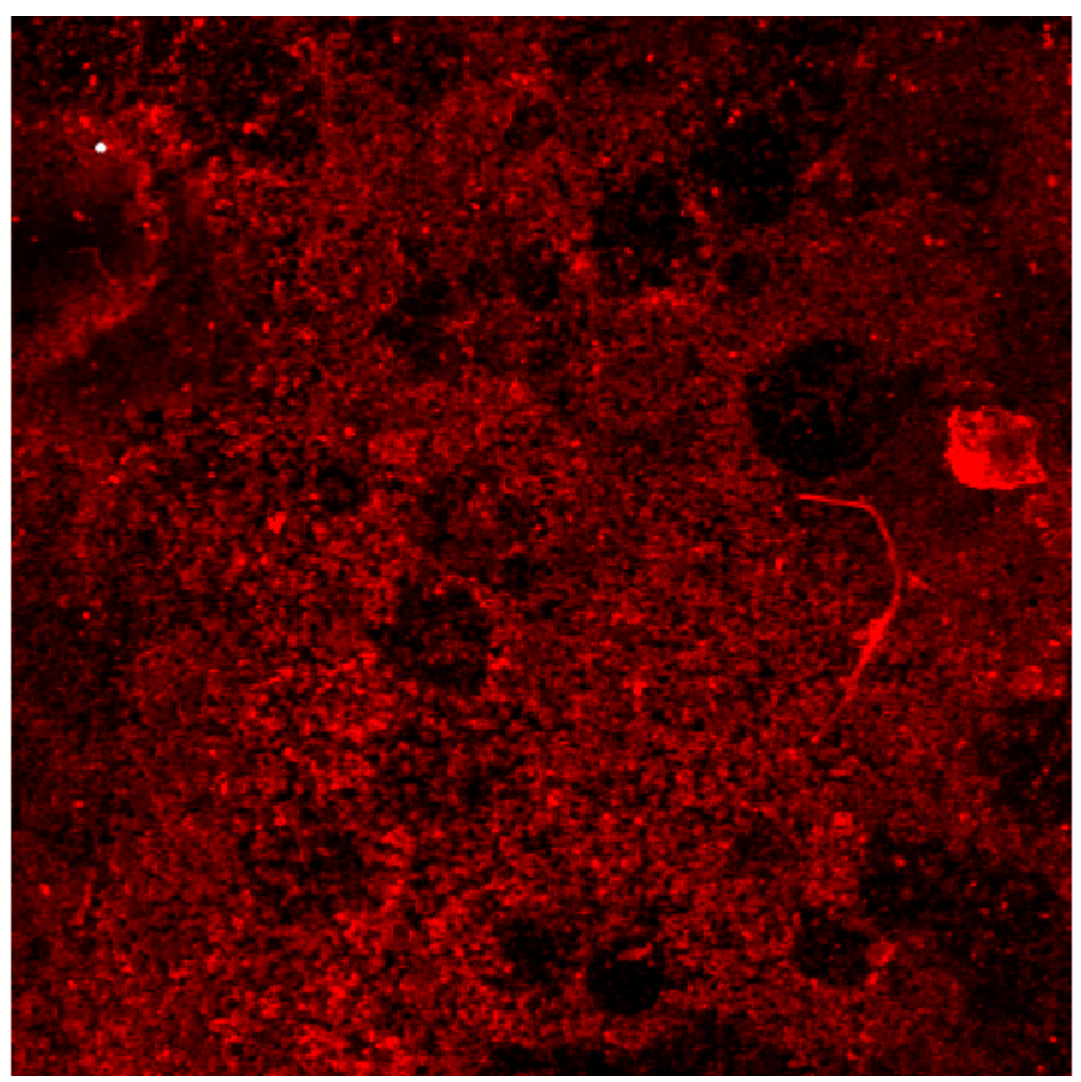

Figure S8. XFM microprobe map (3.5 $\mathrm{mm}^{2}, 10$ micron pixels) of an Ag-BTC strip after testing a $500 \mathrm{ppb}$ arsenic solution, showing incorporation of arsenic across the surface of the test strip.
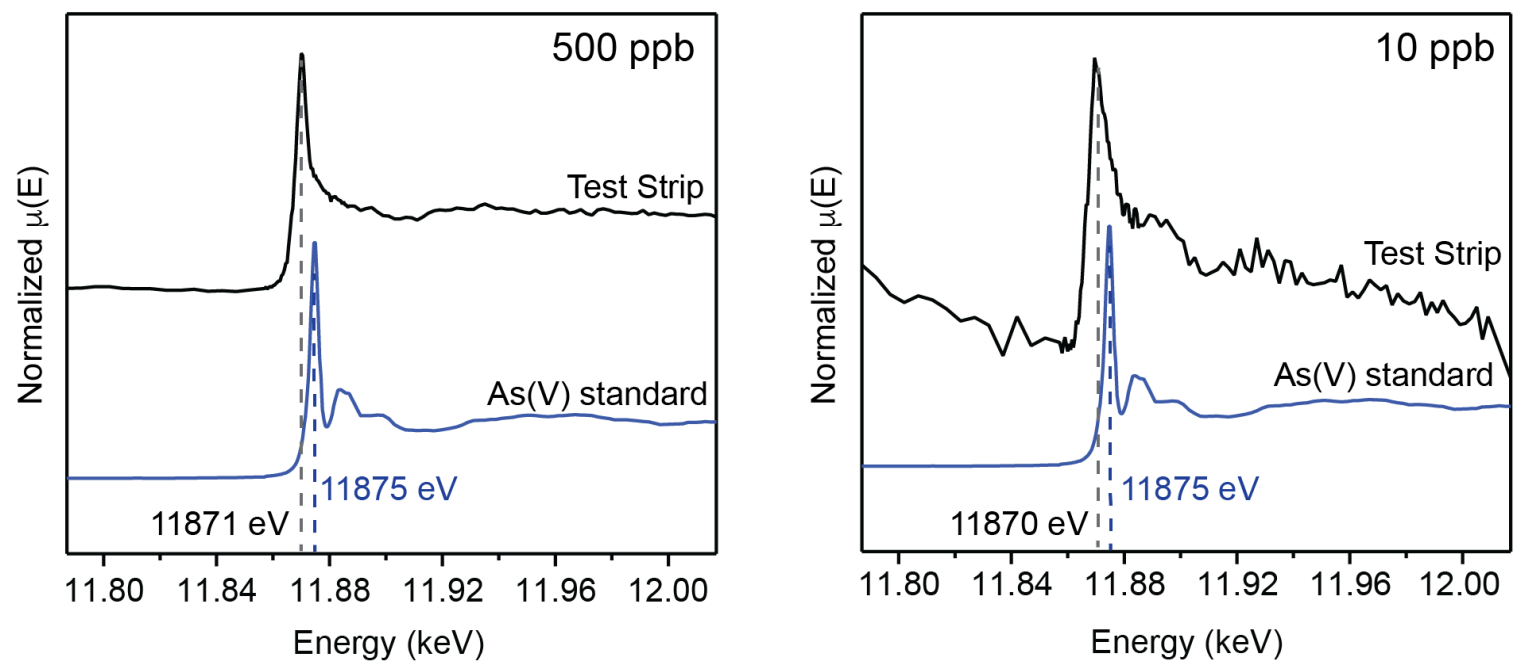

Figure S9. As K-edge XANES data collected for Ag-BTC strips after testing 500 ppb (left) and $10 \mathrm{ppb}$ (right) arsenic solutions, measured with an As(V) standard for reference. The edge positions clearly indicate the presence of arsenic in the +III oxidation state. 


\section{References}

1. Ravel, B.; Newville, M. ATHENA, ARTEMIS, HEPHAESTUS: Data Analysis for X-

Ray Absorption Spectroscopy using IFEFFIT. J. Synchrotron Radiat. 2005, 12, 537541.

2. Webb, S. M. SMAK: Sam's Microprobe Analysis Kit; Stanford Synchrotron Radiation Laboratory, 2006, 1.64

3. Sun, D.; Cao, R.; Bi, W.; Weng, J.; Hong, M.; Liang, Y. Synthesis and characterization of a series of silver-carboxylate polymers Inorg. Chim. Acta 2004, 357, 991-1001.

4. https://www.hach.com/arsenic-low-range-test-kit/product?id=7640217303 (accessed Sept 15, 2021)

5. https://sensafe.com/quick-arsenic-econo/ (accessed Sept 15, 2021)

6. https://sensafe.com/content/481298.pdf (accessed Sept 15, 2021) 